\title{
The Semantics of Predicate Logic as a Programming Language
}

\author{
M. H. VAN EMDEN AND R. A. KOWALSKI \\ University of Edinburgh, Edinburgh, Scotland
}

ABSTRACT Sentences in first-order predicate logic can be usefully interpreted as programs In this paper the operational and fixpoint semantics of predicate logic programs are defined, and the connections with the proof theory and model theory of logic are investigated It is concluded that operational semantics is a part of proof theory and that fixpoint semantics is a special case of model-theoretic semantics

KEY WORDS AND PHRASES predicate logic as a programming language, semantıcs of programmıng languages, resolution theorem proving, operational versus denotational semantics, SL-resolution, fixpoınt charactenzation

CR CATEGORIES* $422,521,524$

\section{Introduction}

Predicate logic plays an important role in many formal models of computer programs [3, $14,17]$. Here we are concerned with the interpretation of predicate logic as a programming language $[5,10]$. The PROLOG system (for PROgramming in LOGic), based upon the procedural interpretation, has been used for several ambitious programming tasks (including French language question answering [5, 18], symbolic integration [9], plan formation [24], theorem proving, speech recognition, and picture interpretation). In this paper we ignore the practical aspects of programming in logic and investigate instead the semantics of predicate logic regarded as a programming language. We compare the resultıng semantics with the classical semantics studied by logicians.

Two kinds of semantics [22], operatıonal and fixpoint, have been defined for programming languages. Operational semantics defines the input-output relation computed by a program in terms of the individual operations evoked by the program inside a machine. The meaning of a program is the input-output relation obtained by executing the program on the machine. As a machine independent alternative to operational semantics, fixpoint semantics $[1,6,17,22]$ defines the meanıng of a program to be the inputoutput relation which is the minımal fixpoint of a transformation associated with the program. Fixpoint semantics has been used $[6,7,15,17]$ to justify existing methods for proving properties of programs and to motivate and justify new methods of proof.

Logicians distinguish between the syntax and the semantics of formal languages. Syntax deals with the formal part of language in abstraction from its meaning. It deals not only with the definition of well-formed formulas, syntax in its narrow sense, but also with the broader study of axioms, rules of inference, and proofs, which constitutes proof theory. Semantics, on the other hand, deals with the interpretation of language and includes such notions as meaning, logical implication, and truth. Church's Introduction to Mathematical Logıc [4] contains a thorough discussion of the respective roles of syntax and semantics.

Copyright $\mathbb{C}$ 1976, Association for Computıng Machınery, Inc General permission to republısh, but not for profit, all or part of this material is granted provided that ACM's copyright notice is given and that reference is made to the publication, to its date of issue, and to the fact that reprinting privileges were granted by permission of the Association for Computing Machinery

This work was supported by the U K Science Research Council

Authors' present addresses M H van Emden, Department of Computer Science, University of Waterloo, Waterloo, Ontario, Canada N2L 3G1, R A Kowalsk1, Department of Computation \& Control, Imperial College, 180 Queens Gate, London SW7, Unıted Kıngdom 
We use the interpretation of predicate logic as a programming language in order to compare the notions of operatıonal and fixpoint semantics of programming languages with the notions of syntax and semantics of predicate logic. We show that operational semantics is included in the part of syntax concerned with proof theory and that fixpoint semantics is a special case of model-theoretic semantics. With this interpretation of operational semantics as syntax and fixpoint semantics as semantics, the equivalence of operational and fixpoint semantics becomes a special case of Godel's completeness theorem.

This paper is concerned with the analysis and comparison of some of the most basic notions of logic and computation As a by-product it is virtually self-contained and requires only a general knowledge of logic but no special familiarity with the operational and fixpoint semantics of programming languages.

\section{A Syntax of Well-Formed Formulas}

It is convenient to restrict attention to predicate logic programs written in clausal form. Such programs have an especially simple syntax but retain all the expressive power of the full predicate logic.

A sentence is a finite set of clauses.

A clause is a disjunction $L_{1} \vee \cdots \vee L_{n}$ of literals $L_{\imath}$, which are atomic formulas $P\left(t_{1}, \ldots, t_{m}\right)$ or the negations of atomic formulas $P\left(t_{1}, \ldots, t_{m}\right)$, where $P$ is a predicate symbol and $t_{2}$ are terms. Atomic formulas are positive literals. Negations of atomic formulas are negative literals.

A term is either a variable or an expression $f\left(t_{1}, \ldots, t_{m}\right)$ where $f$ is a function symbol and $t_{\imath}$ are terms. Constants are 0 -ary function symbols.

A set of clauses $\left\{C_{1}, \ldots, C_{n}\right\}$ is interpreted as the conjunction, $C_{1}$ and $\ldots$ and $C_{n}$. A clause $C$ containing just the variables $x_{1}, \ldots, x_{m}$ is regarded as universally quantıfied:

$$
\text { for all } x_{1}, \ldots, x_{m} C
$$

For every sentence $S_{1}$ in predicate logic there exists a sentence $S_{2}$ in clausal form which is satısfiable if and only if $S_{1}$ is. For this reason, all questıons concernıng the validity or satisfiability of sentences in predicate logic can be addressed to sentences in clausal form. Methods for transforming sentences into clausal form are described in [16].

We have defined that part of the syntax of predicate logic which is concerned with the specification of well-formed formulas. Aspects of syntax concerned with proof theory are dealt with in the next two sections.

\section{The Procedural Interpretation}

It is easiest to interpret procedurally sets of clauses which contain at most one positive literal per clause. Such sets of clauses are called Horn sentences. We distinguish three kinds of Horn clauses.

(1) $\square \quad$ the empty clause, containing no literals and denoting the truth value false, is interpreted as a halt statement.

(2) $\bar{B}_{1} \vee \cdots \vee \bar{B}_{n}$ a clause consistıng of no positıve literals and $n \geq 1$ negatıve literals is interpreted as a goal statement.

(3) $A \vee \bar{B}_{1} \vee \cdots \vee \bar{B}_{n}$ a clause consisting of exactly one positive literal and $n \geq 0$ negative literals is interpreted as a procedure declaration. The positive literal $A$ is the procedure name and the negative literals are the procedure body. Each negative literal $\bar{B}_{\imath}$ in the procedure body is interpreted as a procedure call. When $n=0$ the procedure declaration has an empty body and is interpreted as an unqualified assertion of fact

In the procedural interpretation a set of procedure declarations is a program. Computation is initıated by an initial goal statement, proceeds by using procedure declarations 
to derive new goal statements from old goal statements, and terminates with the derivation of the halt statement Such derivation of goal statements is accomplished by resolution [20], which is interpreted as procedure invocation. Given a selected procedure call $\bar{A}_{\imath}$ inside the body of a goal statement

$$
\bar{A}_{1} \vee \cdots \vee \bar{A}_{\imath-1} \vee \bar{A}_{1} \vee \bar{A}_{1+1} \vee \cdots \vee \bar{A}_{n}
$$

and given a procedure declaration

$$
A^{\prime} \vee \bar{B}_{1} \vee \cdots \vee \bar{B}_{m}, \quad m \geq 0
$$

whose name matches the selected procedure call (1n the sense that some most general substitution $\theta$ of terms for variables makes $A_{\imath}$ and $A^{\prime}$ identical), resolution derives the new goal statement

$$
\left(\bar{A}_{1} \vee \cdots \vee \bar{A}_{t-1} \vee \bar{B}_{1} \vee \cdots \vee \bar{B}_{m} \vee \bar{A}_{1+1} \vee \cdots \vee \bar{A}_{n}\right) \theta
$$

In general, any derivation can be regarded as a computation and any refutation (i.e. derivation of $\square$ ) can be regarded as a successfully terminating computation. However only goal oriented resolution derivations correspond to the standard notion of computation. Such a goal-oriented derivation from an initial set of Horn clauses $\mathbf{A}$ and from an initial goal statement $C_{1}$ in $\mathbf{A}$ is a sequence of goal statements $C_{1}, \ldots, C_{n}$ such that each $C_{\imath}$ contains a single selected procedure call and $C_{\imath+1}$ is obtained from $C_{\imath}$ by procedure invocation relative to the selected procedure call in $C_{2}$ using a procedure declaration in A

In model elımination [13], ordered linear resolution [19], and SL-resolution [12], the selection of procedure calls is governed by the last in/first out rule: A goal statement is treated as a stack of procedure calls. The selected procedure call must be at the top of the stack. The new procedure calls which by procedure invocation replace the selected procedure call are inserted at the top of the stack. The more general notion of goal oriented derivation defined above corresponds to computation with coroutines [10]. Computation with asynchronous parallel processes is obtained by using the splitting rule. $[2,8,23]$.

Predicate logic is a nondeterministic programming language: Given a single goal statement, several procedure declarations can have a name which matches the selected procedure call. Each declaration gives rise to a new goal statement. A proof procedure which sequences the generation of derivations in the search for a refutation behaves as an interpreter for the program incorporated in the initial set of clauses. These and other aspects of the procedural interpretation of Horn clauses are investigated in greater detall elsewhere [10]

The procedural interpretation has also been investigated for non-Horn clauses [11]. However, in this paper we restrict ourselves to Horn clauses.

Example The following two clauses constitute a program for appending two lists. The term cons $(x, y)$ is interpreted as a list whose first element, the head, is $x$ and whose tall, $y$, is the rest of the list. The constant nul denotes the empty list. The terms $u, x, y$, and $z$ are variables. Append $(x, y, z)$ denotes the relationshıp: $z$ is obtained by appending $y$ to $x$.

(1) Appen $(\mathrm{n} 1 \mathrm{l}, x, x)$.

(2) Append(cons $(x, y), z, \operatorname{cons}(x, u)) \vee \overline{\operatorname{Append}}(y, z, u)$

To compute the result of appending the list $\operatorname{cons}(b$, nil $)$ to the list $\operatorname{cons}(a$, nil $)$, the program is activated by the goal statement

(3) $\overline{\text { Append }}(\operatorname{cons}(a, \mathrm{nil}), \operatorname{cons}(b, \mathrm{nil}), v)$,

where $v$ is a variable and $a$ and $b$ are constants, the "atoms" of the lists With this goal statement the program is deterministic. With a goal directed theorem prover as interpreter, the following computation ensues:

$$
\begin{aligned}
& C_{1}=\overline{\operatorname{Append}}(\operatorname{cons}(a, \mathrm{nil}), \operatorname{cons}(b, \mathrm{nil}), v), \\
& C_{2}=\overline{\operatorname{Append}}(\mathrm{nil}, \operatorname{cons}(b, \mathrm{nil}), w) \theta_{1}, \\
& C_{3}=\square \theta_{2},
\end{aligned}
$$


where $\theta_{1}$ is the substitution $v=\operatorname{cons}(a, w)$ and $\theta_{2}$ is $w:=\operatorname{cons}(b$, nil $)$. The result of the computation is the value of $v$ in the substıtution $\theta_{1} \theta_{2}$, which is $v:=\operatorname{cons}(a, \operatorname{cons}(b, \mathrm{n} 1))$.

\section{Operational Semantıcs}

To define an operational semantıcs [22] for a programming language is to define an implementation independent interpreter for it For predicate logic the proof procedure behaves as such an interpreter

We regard the terms containing no variables which can be constructed from the constants and other function symbols occurring in a set of clauses $\mathbf{A}$ as the data structures which the program, incorporated in $\mathbf{A}$, manipulates. The set of all such terms is called the Herbrand universe $H$ determined by $\mathbf{A}$. Every $n$-ary predicate symbol $P$ occurring in $\mathbf{A}$ denotes an $n$-ary relation over the Herbrand universe of $\mathbf{A}$ We call the $n$-tuples which belong to such relations input-output tuples and the relations themselves input-output relations.

Given a specific inference system, the operational semantics determines a unique denotation for $P$ : The $n$-tuple $\left(t_{1}, \ldots, t_{m}\right)$ belongs to the denotation of $P$ in $\mathbf{A}$ iff $\mathbf{A} \vdash$ $P\left(t_{1}, \ldots, t_{n}\right)$, where $X \vdash Y$ means that there exists a derivation of $Y$ from $X$. For resolution systems we employ the convention that $X \vdash Y$ means that there exists a refutation of the sentence in clausal form corresponding to $X \& \bar{Y}$. We use the notation

$$
\mathbf{D}_{1}(P)=\left\{\left(t_{1}, \ldots, t_{n}\right): \mathbf{A} \vdash P\left(t_{1}, \ldots, t_{n}\right)\right\}
$$

for the denotation of $P$ in $\mathbf{A}$ as determined by operational semantics

It needs to be emphasized that only goal oriented inference systems correspond to the standard notion of operational semantics, where procedure calls are replaced by procedure bodies. In theory, however, any inference system for predicate logic specifies, implicitly at least, an abstract machine which generates exactly those derivations which are determined by the given inference system

Notice that in our treatment predicate logic programs compute relations. The relations computed are denoted by predicate symbols in the defining set of clauses $\mathbf{A}$. Those special relations which are functions are also denoted by predicate symbols The function symbols occurring in $\mathbf{A}$ do not denote functions computed by the program but construct the data structures which are the input and output objects of the relations (or functions) computed.

It is a significant application of the proof theory of resolution systems to the computation theory of predicate logic programs that if $\mathbf{A}$ is consistent and $\mathbf{A} \vdash P\left(t_{1}, \ldots, t_{n}\right)$ then there exists a resolution refutation of $\mathbf{A} \& \bar{P}\left(x_{1}, \ldots, x_{n}\right)$ in which the variables $x_{1}, \ldots, x_{n}$ are eventually instantiated to terms which have $t_{1}, \ldots, t_{n}$ as an instance. More generally, if $\mathbf{A} \vdash P\left(t_{1}, \ldots, t_{n}\right)$, then for any subset of the arguments $t_{1}, \ldots, t_{n}$ of $P$ there exists a computation which accepts those arguments of $P$ as input and computes the remaining arguments as output. A useful practical consequence of this fact is that a predicate logic program can first be written to test that a given relationship holds among the members of an $n$-tuple of objects but can later be used to generate, from some subset of objects in the $n$-tuple given as input, the remaining objects in the $n$-tuple as output. See, for example, the goal statement $3(\mathrm{a})$ below. Another important consequence is that variables occurring in input or output can be used to represent incompletely specified data See, for example, the goal statement $3(\mathrm{~b})$ below. It is these considerations which motivate the terminology "input-output relation" for the relation denoted by a predicate symbol in a set of clauses.

Given a consistent set of clauses $\mathbf{A}$ representing a program and given a goal statement $C$, the Herbrand universe for $\mathbf{A}$ can be different from the Herbrand universe for the set of clauses $\mathbf{A} \cup\{C\}$. Although this is an interesting case to consider, we assume for simplicity that it does not arise and that $C$ contains only constant symbols and function symbols occurring in $\mathbf{A}$. Similarly we assume that $\mathbf{A}$ always contains at least one constant symbol. 
Example. The program for appending lists can be activated by the goal statement:

(3a) Append $(x, \operatorname{cons}(a, y), \operatorname{cons}(a, \operatorname{cons}(b, \operatorname{cons}(a, \operatorname{nil}))))$,

where $a, b$, and nll are constants, and $x$ and $y$ are variables. With this goal statement the program behaves nondeterministically: There are two computations, one ends with $x:=$ nil, $y:=\operatorname{cons}(b, \operatorname{cons}(a$, nil $))$, and the other ends with $x:=\operatorname{cons}(a, \operatorname{cons}(b$, nil $)), y:=$ nil. Activated by a goal statement with this pattern of constants and variables, the program checks whether a particular item occurs in the given list and gives a different computation for each different occurrence. For each occurrence of the item, it determines the list of items preceding the given occurrence as well as the list following it.

Example. The program for appending can also be activated by the goal statement:

(3b) Append (cons $(b, \mathrm{nll}), y, z)$,

where $b$ and nil are constants and $y$ and $z$ are variables. Starting from this goal statement there is one computation. It ends with $z:=\operatorname{cons}(b, y)$, which can be interpreted as stating that $z$ is the list whose head is $b$ and whose tail is the unspecified input $y$.

\section{Model-Theorettc Semantıcs}

There is general agreement among logicians concernıng the semantics of predicate logic. This semantics provides a simple method for determining the denotation of a predicate symbol $P$ in a set of clauses $\mathbf{A}$ :

$$
\mathbf{D}_{2}(P)=\left\{\left(t_{1}, \ldots, t_{n}\right): \mathbf{A}=P\left(t_{1}, \ldots, t_{n}\right)\right\},
$$

where $X \vDash Y$ means that $X$ logically implies $Y . \mathbf{D}_{2}(P)$ is the denotation of $\mathbf{P}$ as determined by model-theoretic semantics.

The completeness of first-order logic means that there exist inference systems such that derivability coincides with logical implication; i.e. for such inference systems $X+Y$ iff $X \mid Y$.

The equivalence of operational and model-theoretic semantics $\dot{\mathbf{D}}_{1}(P)=\mathbf{D}_{2}(P)$ is an immediate consequence of the completeness of the inference system which determines $\mathbf{D}_{1}$.

In order to make a comparison of the fixpoint and model-theoretic semantics, we need a more detailed definition of $\mathbf{D}_{2}$. For this purpose we define the notions of Herbrand interpretation and Herbrand model.

An expression (term, literal, clause, set of clauses) is ground if it contans no variables. The set of all ground atomic formulas $P\left(t_{1}, \ldots, t_{n}\right)$, where $P$ occurs in the set of clauses $\mathbf{A}$ and $t_{1}, \ldots, t_{n}$ belong to the Herbrand universe $H$ of $\mathbf{A}$, is called the Herbrand base $\hat{H}$ of A. A Herbrand interpretation $I$ of $\mathbf{A}$ is any subset of the Herbrand base of $\mathbf{A}$. A Herbrand interpretation simultaneously associates, with every $n$-ary predicate symbol in $\mathbf{A}$, a unique $n$-ary relation over $H$. The relation $\left\{\left(t_{1}, \ldots, t_{n}\right): P\left(t_{1}, \ldots, t_{n}\right) \in I\right\}$ is associated by $I$ with the predicate symbol $P$ in $\mathbf{A}$.

(1) A ground atomic formula $A$ is true in a Herbrand interpretation $I$ iff $A \in I$.

(2) A ground negative literal $\bar{A}$ is true in $I$ iff $A \notin I$.

(3) A ground clause $L_{1} \vee \cdots \vee L_{m}$ is true in $I$ iff at least one literal $L_{\imath}$ is true in $I$.

(4) In general a clause $C$ is true in $I$ iff every ground instance $C \sigma$ of $C$ is true in $I$. ( $C \sigma$ is obtained by replacing every occurrence of a variable in $C$ by a term in $H$. Different occurrences of the same variable are replaced by the same term.)

(5) A set of clauses $\mathbf{A}$ is true in $I$ iff each clause in $\mathbf{A}$ is true in $I$.

A literal, clause, or set of clauses is false in $I$ iff it is not true. If $\mathbf{A}$ is true in $I$, then we say that $I$ is a Herbrand model of $\mathbf{A}$ and we write $F_{I} \mathbf{A}$. It is a simple version of the Skolem-Lowenheim theorem that a sentence $\mathbf{A}$ in clausal form has a model iff it has a Herbrand model.

We can now formulate an explicit definition of the denotation determined by the model-theoretic semantics. Let $\mathbf{M}(\mathbf{A})$ be the set of all Herbrand models of $\mathbf{A}$; then $\cap \mathbf{M}(\mathbf{A})$, the intersection of all Herbrand models of $\mathbf{A}$, is itself a Herbrand interpretation 
of $\mathbf{A}$. If $\mathbf{A}$ contains the predicate symbol $P$, then the denotation $\mathbf{D}_{2}(P)$ is the relation associated with $P$ by the Herbrand interpretation $\cap \mathbf{M}(\mathbf{A})$. In symbols,

$$
\mathbf{D}_{2}(P)=\left\{\left(t_{1}, \ldots, t_{n}\right): P\left(t_{1}, \ldots, t_{n}\right) \in \cap \mathbf{M}(\mathbf{A})\right\}
$$

for any set of clauses $\mathbf{A}$.

Proof. $\left(t_{1}, \ldots, t_{n}\right) \in \mathbf{D}_{2}(P)$

iff $\mathbf{A}=P\left(t_{1}, \ldots, t_{n}\right)$,

iff $\mathbf{A} \cup\left\{\dot{P}\left(t_{1}, \ldots, t_{n}\right)\right\}$ has no model,

iff $\mathbf{A} \cup\left\{\bar{P}\left(t_{1}, \ldots, t_{n}\right)\right\}$ has no Herbrand model,

iff $\bar{P}\left(t_{1}, \ldots, t_{n}\right)$ is false in all Herbrand models of $\mathbf{A}$,

iff $P\left(t_{1}, \ldots, t_{n}\right)$ is true in all Herbrand models of $\mathbf{A}$,

iff $P\left(t_{1}, \ldots, t_{n}\right) \in \cap \mathbf{M}(\mathbf{A})$.

Notice that the above equality holds for any set of clauses $\mathbf{A}$ even if $\mathbf{A}$ is inconsistent. If $\mathbf{A}$ is a consistent set of Horn clauses then $\cap \mathbf{M}(\mathbf{A})$ is itself a Herbrand model of $\mathbf{A}$. More generally, Horn clauses have the model intersectton property: If $\mathbf{L}$ is any nonempty set of Herbrand models of $\mathbf{A}$ then $\cap \mathbf{L}$ is also a model of $\mathbf{A}$.

Proof. Assume $\cap \mathbf{L}$ is not a model of $\mathbf{A}$. Then $\cap \mathbf{L}$ falsifies some ground instance $C \sigma$ of a clause $C \in \mathbf{A}$.

If $C$ is a procedure declaration, then

$$
C \sigma=A \vee \bar{A}_{1} \vee \cdots \vee \bar{A}_{m}, m \geq \mathbf{0}, \quad A \notin \cap \mathbf{L}, \quad \text { and } A_{1}, \ldots, A_{m} \in \cap \mathbf{L} .
$$

Therefore for some $I \in \mathbf{L}, A \notin I$ and $A_{1}, \ldots, A_{m} \in I . C$ is false in $I$, contrary to assumption that $I \in \mathbf{L}$.

If $C$ is a goal statement, then

$$
C \sigma=\bar{A}_{1} \vee \cdots \vee \bar{A}_{m}, m>0, \quad A_{1}, \ldots, A_{m} \in \cap \mathbf{L} .
$$

Therefore for all $I \in \mathbf{L}, A_{1}, \ldots, A_{m} \in I . C$ is false in $I$, contrary to assumption that $I \in \mathbf{L}$.

$\{P(a) \vee P(b)\}$, where $a$ and $b$ are constants, is an example of a non-Horn sentence which does not have the model-intersection property: $\{\{P(a)\},\{P(b)\}\}$ is a nonempty set of models, yet its intersection $\varnothing$ is a Herbrand interpretation which is not a model.

\section{Fixpoint Semantics}

In the fixpoint semantics, the denotation of a recursively defined procedure is defined to be the minimal fixpoint of a transformation associated with the procedure definition. Here we propose a similar definition of fixpoint semantics for predicate logic programs. In order to justıfy our definıtıon we first describe the fixpoint semantics as it has been formulated for more conventionally defıned recursive procedures. Our description follows the one given by de Bakker [6]

Let $P \Leftarrow \mathbf{B}(P)$ be a procedure declaration in an Algol-like language, where the first occurrence of $P$ is the procedure name, where $\mathbf{B}(P)$ is the procedure body, and where the occurrence of $P$ in $\mathbf{B}(P)$ distinguishes all calls to $P$ in the body of the procedure. Associated with $\mathbf{B}$ is a transformation $T$ which maps sets $I$ of input-output tuples into other such sets $J=T(I)$. When the transformation $T$ is monotonic (which means that $T\left(I_{1}\right) \subseteq T\left(I_{2}\right)$ whenever $\left.I_{1} \subseteq I_{2}\right)$ the denotation of $P$ is defined as

$$
\cap\{I: T(I) \subseteq I\}
$$

which is identical to the intersection of all fixpoints of $T$,

$$
\cap\{I: T(I)=I\} \text {, }
$$

and which is itself a fixpoint (the least such) of $T$.

In a similar way a transformation $T$ can be associated with a finite set of mutually recursive procedure declarations 


$$
\begin{gathered}
P_{1} \Leftarrow \mathbf{B}_{1}\left(P_{1}, \ldots, P_{n}\right) \\
\cdot \\
P_{n} \Leftarrow \mathbf{B}_{n}\left(P_{1}, \ldots, P_{n}\right) .
\end{gathered}
$$

The minimal fixpoint of $T$, which exists when $T$ is monotonic, can be decomposed into components, the $t$ th of which is the denotation of the procedure $P_{\imath}$.

By means of the procedural interpretation, the fixpoint semantics of predicate logic is defined similarly. A set of Horn clauses of the form $A \vee A_{1} \vee \cdots \vee A_{m}$, where $m \geqslant 0$, is interpreted as a set of mutually recursive, possibly nondeterministic, procedure declarations We restrict the definition of the fixpoint semantics of predicate logic programs to sentences $\mathbf{A}$ which are sets of such procedure declarations. Associated with every such sentence $\mathbf{A}$ is a transformation $T$ which maps Herbrand interpretations to Herbrand interpretations. Suppose that $P_{1}, \ldots, P_{n}$ are the predicate symbols occurring in $\mathbf{A}$. The transformation $T$ can be defined in terms of individual transformations $T_{1}$ associated with the individual predicate symbols $P_{1}$. $T_{1}$ maps Herbrand interpretations $I$ to Herbrand interpretations $J_{1}=T_{1}(I)$ which contain only atomic formulas beginning with the predicate symbol $P_{i}$ :

$J_{1}=T_{1}(I)$ contains a ground atomic formula $A \in \hat{H}$ iff $A$ begins with the predicate symbol $P_{\imath}$ and, for some ground instance $C \sigma$ of a clause $C$ in $\mathbf{A}, C \sigma=A \vee \bar{A}_{1}$ $\vee \cdots, \bar{A}_{m}$ and $A_{1}, \ldots, A_{m} \in I, m \geqslant 0$.

The transformation $T$ associated with $\mathbf{A}$ is defined by $T(I)=T_{1}(I) \cup \cdots \cup T_{n}(I)$.

The input-output relation associated by $J_{1}=T_{1}(I)$ with $P_{1}$ can be regarded as the relation obtained by "substituting," for the procedure calls in the declarations of $P_{1}$ in $\mathbf{A}$, the appropriate input-output relations associated by $I$. This interpretation of $T_{2}$ is analogous to the corresponding definition for conventıonally defined recursive procedures. A simpler definition of $T$, which is less directly analogous to the conventional definition, is the following:

$T(I)$ contans a ground atomic formula $A \in \hat{H}$ iff for some ground instance $C \sigma$ of a clause $C$ in $\mathbf{A}, C \sigma=A \vee \bar{A}_{1} \vee \cdots \vee \bar{A}_{m}$ and $A_{1}, \ldots, A_{m} \in I, m \geq 0$.

Notice that, independently of $I, T(I)$ always contains all ground instances $A \sigma$ of unqualified assertions $A$ in A (corresponding to the case $m=0$ in the definition of $T(I)$ ).

Let $\mathbf{C}(\mathbf{A})$ be the set of all Herbrand interpretations closed under the transformation $T$, 1.e. $I \in \mathbf{C}(\mathbf{A})$ iff $T(I) \subseteq I$. The denotation of a predicate symbol $P$ occurring in a set of procedure declarations $\mathbf{A}$, as determined by the fixpoint semantics, is

$$
\mathbf{D}_{3}(P)=\left\{\left(t_{1}, \ldots, t_{n}\right): P\left(t_{1}, \ldots, t_{n}\right) \in \cap \mathbf{C}(\mathbf{A})\right\} .
$$

As a corollary of the theorem below, $\cap \mathbf{C}(\mathbf{A})$ is itself closed under $T$ and therefore $\mathbf{D}_{3}(P)$ is the smallest set of input-output tuples closed under $T$. In conventional fixpoint theory this fact is proved by using the monotonicity of $T$.

\section{Model-Theorettc and Fixpoint Semantics}

We shall show that for sets of procedure declarations $\mathbf{A}$, model-theoretic and fixpoint semantics coincide: $\mathbf{D}_{2}=\mathbf{D}_{3}$. It would be sufficient to show that $\cap \mathbf{M}(\mathbf{A})=\cap \mathbf{C}(\mathbf{A})$, but it is easy to prove that even $\mathbf{M}(\mathbf{A})=\mathbf{C}(\mathbf{A})$.

In other words, a Herbrand interpretation $I$ of $\mathbf{A}$ is a model of $\mathbf{A}$ iff $I$ is closed under the transformation $T$ associated with $\mathbf{A}$.

TheOREM. If $\mathbf{A}$ is a set of procedure declarations, then $\mathbf{M}(\mathbf{A})=\mathbf{C}(\mathbf{A})$, 1.e. $F_{I} \mathbf{A}$ iff $T(I) \subseteq I$, for all Herbrand interpretations 1 of $\mathbf{A}$

Proof. $\quad\left(F_{I} \mathbf{A}\right.$ implies $T(I) \subseteq I$.) Suppose that $I$ is a model of $\mathbf{A}$. We want to show $J=$ $T(I) \subseteq I$, 1.e. that if $A \in J$ then $A \in I$. 
Assume that $A \in J$; then by the definition of $T$, for some $C \in \mathbf{A}$ and for some ground instance $C \sigma$ of $C$,

$$
C \sigma=A \vee \bar{A}_{1} \vee \cdots \vee \bar{A}_{n} \text { and } A_{1}, \ldots, A_{n} \in I .
$$

Because $I$ is a model of $A, C \sigma$ is true in $I$. But then $A$ is true in $I$, because $\bar{A}_{1}, \ldots$, and $\bar{A}_{n}$ are false in $I$. Therefore $A \in I$.

$\left(T(I) \subseteq I\right.$ implies $\left.\models_{I} \mathbf{A}\right)$. Suppose that $I$ is not a model of $\mathbf{A}$. We want to show that $T(I)$ $\nsubseteq \mathbf{I}$. But $I$ falsifies some ground instance $C \sigma$ of a clause $C$ in $\mathbf{A}$, where $C \sigma=A \vee \dot{A}_{1}$ $\vee \cdots \vee \bar{A}_{m}, m \geq 0$. Because $I$ falsifıes $C \sigma, A \notin I$ and $A_{1}, \ldots, A_{m} \in I$. But then, because $A_{1}, \ldots, A_{m} \in I$, it follows that $A \in T(I)$. Therefore $T(I) \nsubseteq \mathrm{I}$.

CoROLlaRY. If $\mathbf{A}$ is a set of procedure declarations, then $\cap \mathbf{C}(\mathbf{A})$ is closed under $T$.

Proof. $\cap \mathbf{C}(\mathbf{A})=\cap \mathbf{M}(\mathbf{A})$ by the model-intersection property is a model of $\mathbf{A}$ and by the theorem is therefore closed under $T$.

\section{Openational and Fixpoint Semantics, Hyperresolution}

The equivalence $\mathbf{D}_{1}=\mathbf{D}_{3}$ between operational and fixpoint semantics, which follows from the equivalences $\mathbf{D}_{1}=\mathbf{D}_{2}$ and $\mathbf{D}_{2}=\mathbf{D}_{3}$, has different interpretations depending upon the inference system which determınes $\mathbf{D}_{1}$. Here we investigate the interpretation associated with a particular inference system based upon hyperresolution [21].

For ground procedure declaratıons the definition of hyperresolution is very simple:

An atomic formula $A$ is the hyperresolvent of ground clauses $A \vee \dot{A} 1 \vee \cdots \vee \bar{A}_{m}$ and $A_{1}, \ldots, A_{m} . A$ is said to be obtained from $A \vee \dot{A}_{1} \vee \cdots \vee \dot{A}_{m}$ and $A_{1}, \ldots, A_{m}$ by hyperresolution.

The connection with fixpoint semantics is obvious: If $T$ is the transformation associated with the set of procedure declarations $\mathbf{A}$ and if $I$ is a Herbrand interpretation of $\mathbf{A}$, then $T(I)$ is the set of all ground instances of assertions in $\mathbf{A}$ together with all hyperresolvents derivable in one step from ground instances of clauses in $\mathbf{A}$ and from assertions in 1 . It follows that

$A$ is derivable by means of a hyperresolution derivation from ground instances of clauses in $\mathbf{A}$ iff $A \in \cup_{m=0}^{\infty} T^{m}(\varnothing)$ where $T^{0}(\varnothing)=\varnothing$ and $T^{m+1}(\varnothing)=T\left(T^{m}(\varnothing)\right.$ ).

Let $\mathbf{D}_{1}^{\text {H }}$ be the operational semantics associated with the two inferences rules of ground instantiation of clauses in $\mathbf{A}$ and ground hyperresolution, i.e define

$$
\left(t_{1}, \ldots, t_{n}\right) \in \mathbf{D}_{1}^{\mathrm{H}}(P) \text { iff } P\left(t_{1}, \ldots, t_{n}\right) \in \bigcup_{m=0}^{\infty} T^{m}(\varnothing) \text {. }
$$

The equivalence of $\mathbf{D}_{1} \mathbf{H}$ and the model-theoretic semantıcs $\mathbf{D}_{2}$ is the completeness, for Horn clauses, of the inference system whose inference rules are ground instantiation of input clauses and ground hyperresolution. Completeness can be proved using standard resolution-theoretic arguments. Here we present an alternative direct proof that for any set of declarations $A$ with associated transformation $T, \cup_{m=0}^{\infty} T^{m}(\varnothing)=\cap M(A)$.

Proof. Let $\mathbf{U}$ abbreviate $\mathbf{U}_{m=0}^{\infty} T^{m}(\varnothing)$.

( $\mathbf{U} \subseteq \cap \mathbf{M}(\mathbf{A})$ ). Suppose that $A \in \mathbf{U}$. Then $A$ is derivable by means of a hyperresolution derivation from ground instances of clauses in $\mathbf{A}$. By the correctness of hyperresolution and instantiation, $\mathbf{A} \vDash A$ and therefore $A \in \cap \mathbf{M}(\mathbf{A})$.

$(\cap \mathbf{M}(\mathbf{A}) \subseteq \mathbf{U})$. We show that $\mathbf{U}$ is closed under $T$, because then $\mathbf{U} \in \mathbf{M}(\mathbf{A})$, and therefore $\cap \mathbf{M}(\mathbf{A}) \subseteq \mathbf{U}$. Suppose that $A \in T(\mathbf{U})$. By the definition of $T$, either $A$ is an instance of an unqualified assertion in $\mathbf{A}$ or some clause $A \vee \bar{A}_{1} \vee \cdots \vee \bar{A}_{n}$ is an instance of a clause in $\mathbf{A}$ and $A 1, \ldots, A_{n} \in \mathbf{U}$. In the first case $A \in \mathbf{U}$, because $A \in T^{m}(\varnothing), m>0$. In the second case $A_{1}, \ldots, A_{n} \in T^{N}(\varnothing)$ for some $N \geq 0$, and therefore $A \in T^{N+1}(\varnothing)$ and $A \in \mathbf{U}$. Therefore $\mathbf{U}$ is closed under $T$.

Therefore for sets of declarations, $\mathbf{D}_{\mathbf{1}}^{\mathbf{H}}=\mathbf{D}_{\mathbf{2}}$.

Because of the equivalence between model-theoretic and fixpoint semantics, we also have that $D_{1}^{H}=D_{3}$, i.e. $U_{m=0}^{\infty} T^{m}(\varnothing)=\bigcap\{I: T(I) \subseteq I\}$. 
This last fact is usually proved in the fixpoint theory by demonstrating the continuity ot the transformation $T$.

\section{Conclusion}

For arbitrary sentences $X$ and $Y$ of first-order predicate logic, proof theory determines when $X \vdash Y$ and model theory determınes when $X \vDash Y$. We have argued that in the procedural interpretation, operational semantics is proof theory and fixpoint semantics is model theory. On the other hand, operational and fixpoint semantics only deal with the case where $Y$ is a set of ground atomic formulas. Moreover, fixpoint semantics only deals with $X$, a set of procedure declarations. We believe that the added generality of proof theory and model theory has useful consequences.

The completeness theorem of first-order logic states that the relations $\vdash$ of derivability and $k$ of logical implication are equivalent. For goal oriented inference systems this equivalence establishes that various computation rules compute the relation determined by the fixpoint semantics. More generally, this equivalence can be used to justify various rules (such as Scott's induction rule [6]) for proving properties of programs.

We have argued that various notions of the conventional theory of computing can be understood in terms of the classical theory of predicate logic. We believe moreover that the predicate logic theory has further contributions to make both to the theory and to the practice of computing.

ACKNOWLEDgments. We are indebted to Michael Gordon for his interest and useful criticism of work leading to this paper. Thanks are due also to Keith Clark, Alain Colmerauer, Gerard Huet, David Park, and Willem-Paul de Roever for their helpful comments on earlier versions of the paper. Suggestions from the referees have also been incorporated in the paper.

\section{REFERENCES}

1 Bekić, H Definable operations in general algebra, and the theory of automata and flow charts IBM Res Rep, Vienna, 1971

2 Bledsoe, W W Splitting and reduction heuristics in automatic theorem proving Artif Intel 2 (1971), $55-77$

3 Burstall, R M Formal description of program structure and semantics in first order logic. In Machine Intelligence 5, B Meltzer and D Michie, Eds, Edınburgh U Press, Edinburgh, 1969, pp 79-98

4 Church, A Introduction to Mathemattcal Logic, Vol 1 Princeton U Press, Princeton, N J , 1956

5 Colmerauer, A , Kanoui, H , Pasero, R., And Roussel, P. Un système de communication hommemachıne en françaıs. Groupe d'Intellıgence Artıficıelle, U E R de Lumıny, Unıversıté d'Aıx-Marseılle, Lumıny, 1972

6 DE BAKKer, J W Recursive procedures Tract No 24, Mathematical Centre, Amsterdam, 1971

7 DE BAKKer, J W , AND DE Roever. W P A calculus of recursive program schemes. In Automata, Languages and Programming, M Nivat, Ed, North-Holland Pub Co, Amsterdam, 1973, pp 167196

8 ERNST, G W The utility of independent subgoals in theorem proving Inform Contr 18, 3 (April 1971), 237-252

9 KANOUı, H Applıcatıon de la démonstratıon automatıque aux manıpulatıons algebrıques et à l'ıntégratıon formelle sur ordınateur Groupe d'Intelligence Artıfıcielle, UE R de Lumıny, Unıversité d'AıxMarseille, Lumıny, 1973.

10 Kowalski, R Predicate logic as programming language Proc IFIP Cong 1974, North-Holland Pub Co, Amsterdam, 1974, pp 569-574

1 Kowalski, R Logic for problem-solving DCL Memo 75, Dep Artıficial Intelligence, U. of Edınburgh, Edinburgh, 1974

12 KowAlSKI, R , AND KUehNer, D Linear resolution with selection function Artif Intel 2 (1971), 227 260

13. Loveland, D.W A simpified format for the model elımination theorem-proving procedure. $J, A C M$ 16, 3 (July 1969), 349-363

14 Manna, Z Properties of programs and the first-order predicate calculus $J A C M 16,2$ (April 1969), 244-255

15 Milner, $R$ Implementation and applications of Scott's logic for computable functions. Proc. ACM Conf on Proving Assertions About Programs, Jan 1972, pp 1-6 
16. NiLsson, N J Problem Solving Methods in Artificial Intelligence. McGraw-Hıll, New York, 1971

17 PARK, D Fixpoint induction and proofs of program properties In Machune Intelligence 5, B Meltzer and D Michie, Eds, Edınburgh U Press, Edınburgh, 1969, pp 59-78

18 Paséro, R. Représentation du françaıs en logıque du premier ordre en vue de dıaloguer avec un ordınateur Group d'Intelligence Artıfıcıelle, U.E R de Lumıny, Unıversıté d'Aıx-Marseılle, Lumıny, 1973.

19 Reiter, $\mathrm{R}$ Two results on ordering for resolution with merging and linear format $J A C M 18,4$ (Oct 1971), 630-646

20. Robinson, J A A machıne-oriented logic based on the resolution principle. J ACM 12, 1 (Jan. 1965), 23-41.

21 Robinson, J A Automatic deduction with hyper-resolution Int J Comptr Math 1 (1965), 227-234

22 Scotr, D Outlıne of a mathematical theory of computation Tech Monog PRG-2, Comptg Lab, Oxford U, Oxford, England

23 S LAGLE, J R , AND Koniver, P Finding resolution graphs and using duplicate goals in AND/OR trees Inform Scl 3 (1971), 315-342

24 WARREN, D H D WARPLAN A system for generating plans DCL Memo 76, Dep. of Artıficial Intelligence, $U$ of Edinburgh, Edınburgh, 1974

RECEIVED MARCH 1974, REVISED APRIL 1976 